\title{
O crime de tráfico de pessoas como hipótese de extradição entre o Brasil e os países
}

\section{da América do Sul}

The crime of human trafficking as an extradition hypothesis between Brazil and the South

\author{
American countries
}

El delito de trata de personas como hipótesis de extradición entre Brasil y países sudamericanos

Recebido: 25/12/2021 | Revisado: 31/12/2021 | Aceito: 10/01/2022| Publicado: 12/01/2022

\author{
Jordana Martins Perussi \\ ORCID: https://orcid.org/0000-0001-8413-5057 \\ Universidade Estadual Paulista, Brasil \\ E-mail:jordanamp21@gmail.com \\ Paulo César Corrêa Borges \\ ORCID: https://orcid.org/0000-0002-5170-7271 \\ Universidade Estadual Paulista, Brasil \\ E-mail: paulo.c.c.borges@unesp.br
}

\begin{abstract}
Resumo
O tráfico de pessoas caracteriza uma grave violação dos direitos humanos, na medida que configura uma forma contemporânea de escravidão, enquanto transforma suas vítimas em mercadorias. A pesquisa buscou pormenorizar esse crime à luz do Protocolo de Palermo, e inferir a influência que a política de enfrentamento ao tráfico de pessoas causou no âmbito das extradições. A partir da publicação daquele protocolo, gradativamente, ocorreu uma uniformização da legislação sul-americana, expandindo o entendimento das diversas formas que assume o tráfico de pessoas. Partindo-se da hipótese que essa homogeneização trazida pelo Protocolo de Palermo poderia implicar na facilitação das extradições de traficantes de pessoas, foi realizada uma pesquisa empírica dialética, com pesquisa documental no Supremo Tribunal Federal brasileiro e nas diversas Cortes Supremas da América do Sul. Nota-se, em um âmbito geral, a existência de uma escassez jurisprudencial. Todavia, foi possível constatar que as extradições foram respaldadas nos acordos bilaterais de extradição entre os países envolvidos e, desse modo, o Protocolo de Palermo não era mencionado de maneira explícita no deferimento das solicitações de extradição. Apesar disso, à exceção da República Bolivariana da Venezuela, todos os países sul-americanos possuem uma legislação do crime abrangendo todas as formas indicadas no Protocolo de Palermo. Portanto, tem-se que a homogeneização contribuiu para o deferimento das extradições, pois um dos prérequisitos acordados nos tratados bilaterais era justamente a dupla incriminação. Assim, se todos os países abrangem as mesmas concepções de tráfico de pessoas em suas legislações, o requisito da dupla incriminação sempre será preenchido.
\end{abstract}

Palavras-chave: Extradição; Tráfico de pessoas; América do Sul; Protocolo de Palermo.

\begin{abstract}
Trafficking in persons features a severe violation of Human Rights whereas sets up a contemporaneous mode of slavery as turn your victims into goods. This research seeks to detail this crime by the light of Palermo Protocol and understand the influence brought by this politics of fighting trafficking in persons caused in the extraditions environment. Starting with the publication of this Protocol, slowly, there has been a standardization of the south American legislation, enlarging the knowledge of the various shapes that human trafficking can assume. Starting with the hypothesis that this standardization brought by the Protocol could mean the facilitation of the extradition, it was performed as an empirical dialectical research, along with documental research in the Brazilian Supremo Tribunal Federal and in the other south American Supreme Courts. The scarcity of jurisprudence is notable. However it was possible to realize that the extraditions were on the bilateral conventions, thus, the Palermo Protocol wasn't explicitly mentioned on the granting of the extradition demands. Besides that, except the Bolivarian Republic of Venezuela, all the south American countries have a legislation on human trafficking including all shapes of this crime as contained in the Protocol. The conclusion from this information is that the standardization has contributed to the granting of the extradition requests, because one of the requirements in the bilateral conventions is the need for double incrimination. Therefore, if all the countries have the same conceptions of human trafficking in their legislations, the requirement of double incrimination will always be filled.
\end{abstract}

Keywords: Extradition; Trafficking in persons; South America; Palermo Protocol. 


\begin{abstract}
Resumen
La trata de personas caracteriza una grave violación de los derechos humanos, ya que configura una forma contemporánea de esclavitud, al tiempo que transforma a sus víctimas en mercancías. La investigación buscó detallar este delito a la luz del Protocolo de Palermo, e inferir la influencia que tuvo la política de combate a la trata de personas en el contexto de las extradiciones. A partir de la publicación de ese protocolo, paulatinamente, se fue homologando la legislación sudamericana, ampliando el entendimiento de las diferentes formas que adopta la trata de personas. Partiendo de la hipótesis de que esta homogeneización provocada por el Protocolo de Palermo podría implicar en la facilitación de extradiciones de traficantes de personas, se realizó una investigación empírica dialéctica, con investigación documental en la Corte Suprema Federal de Brasil y en las distintas Cortes Supremas de América del Sur. Nótese en un ámbito general, la existencia de una falta de jurisprudencia. Sin embargo, se encontró que las extradiciones fueron respaldadas por acuerdos bilaterales de extradición entre los países involucrados y, por lo tanto, el Protocolo de Palermo no fue mencionado explícitamente en el otorgamiento de solicitudes de extradición. A pesar de ello, con excepción de la República Bolivariana de Venezuela, todos los países de América del Sur cuentan con legislación penal que abarca todas las formas señaladas en el Protocolo de Palermo. Por tanto, la homogeneización ha contribuido al otorgamiento de extradiciones, ya que uno de los requisitos previos pactados en los tratados bilaterales fue precisamente la doble incriminación. Por tanto, si todos los países contemplan las mismas concepciones de la trata de personas en su legislación, siempre se cumplirá el requisito de la doble incriminación.
\end{abstract}

Palabras clave: Extradición; Trata de personas; América del Sur; Protocolo de Palermo.

\title{
1. Introdução
}

O tráfico de pessoas configura uma grave violação dos direitos humanos, contudo, trata-se de um delito com pouca visibilidade, na medida em que as redes responsáveis por esse delito atuam de maneira furtiva, tornando a hipótese de quantificar e mapear a real incidência desse crime, uma tarefa árdua. De acordo com Rodrigues (2012), esse tipo penal possui ligação direta com a globalização, posto que esse fenômeno tornou o tráfico um negócio lucrativo, e fornece aos traficantes de pessoas acesso a vítimas em situação de vulnerabilidade, suscetíveis a serem coagidas ou enganadas para serem posteriormente exploradas. Segundo dados da Organização Internacional do Trabalho (OIT), estima-se que durante o ano de 2005, o tráfico de pessoas produziu 2,4 milhões de vítimas, e o lucro anual produzido chegou a 31,6 bilhões de dólares.

No que tange à sub-região da América do Sul, é possível observar a amplitude desse crime através das informações contidas no "Global Report on Trafficking in Persons", relatório produzido e publicado pelo Escritório das Nações Unidas. Através dele foi possível constatar que foram reportadas 3.421 vítimas durante o ano de 2018. Contudo, tais números não são refletidos de maneira equivalente nas condenações de traficantes de pessoas, posto que, neste mesmo recorte temporal, apenas 149 pessoas foram condenadas por praticarem tráfico humano, e destes, foi apurado que apenas $16 \%$ dos sentenciados em primeira instância pertenciam a outras nações (UNODC, 2020).

Nesse contexto, partindo-se da hipótese de que as disposições trazidas no Protocolo das Nações Unidas contra o Crime Organizado Transnacional Relativo à Prevenção, Repressão e Punição do Tráfico de Pessoas, em Especial Mulheres e Crianças causaram uma uniformização na legislação penal referente ao crime de tráfico de pessoas dos países sul-americanos, esse estudo buscou compreender este tratado internacional como ferramenta de cooperação internacional no combate ao crime em tela, e de que maneira ocorreu sua absorção e influência nos ordenamentos jurídicos da América do Sul, especialmente no que tange à possibilidade do seu impacto no deferimento das extradições realizadas entre tais países, referentes ao tráfico humano. Em relação à realidade brasileira, é notável a drástica mudança legislativa ocorrida com a introdução da Lei nº 13.344 de 2016 (Brasil, 2016), a qual adicionou o atual artigo a respeito do crime de tráfico de pessoas no ordenamento jurídico brasileiro, em consonância com a redação prevista no Protocolo de Palermo.

Esse estudo procurou demonstrar, através das análises jurisprudenciais, os métodos de resolução de conflitos internacionais de maneira pacífica, reiterando o interesse e necessidade da cooperação internacional para combater o delito do tráfico de pessoas. Dessa forma, foram apresentadas as disposições concernentes aos tratados bilaterais de extradição firmados entre os países e analisadas as hipóteses de concessão ou recusa de um pedido extradicional. Ademais, são apresentadas as maiores dificuldades enfrentadas no âmbito do combate ao tráfico de pessoas, assim como a necessidade de realização de medidas 
de combate a essa criminalidade envolvendo uma abordagem multigarantista e multiescalar, existindo portanto, além da necessidade de políticas públicas do Estado e de organizações nacionais e internacionais, a indispensabilidade da cooperação pela sociedade civil.

\section{Metodologia}

O presente estudo consistiu, em um primeiro momento, em uma pesquisa bibliográfica e documental, sendo realizado um levantamento bibliográfico a respeito do crime de tráfico de pessoas, assim como suas finalidades e os elementos normativos constituintes de sua tipicidade penal. Para isso, foram realizadas leituras e análises de importantes doutrinadores, como por exemplo Cezar Roberto Bitencourt (2021), Guilherme de Souza Nucci (2020), Rogério Greco (2019), Núria Cordero e Pilar Cruz Zuniga (2019) e Paulo César Corrêa Borges (2013; 2017; e 2019).

Ato contínuo foram analisadas fontes indiretas reiterando a prática do crime de tráfico de pessoas na região da América do Sul. Nesse momento foram compiladas pesquisas jurisprudenciais, realizadas principalmente através da ferramenta Sherloc do Escritório das Nações Unidas, indicando casos ocorridos do delito em questão em cada um dos países sul-americanos, especialmente na modalidade transfronteiriça, a fim de analisar o processo de cooperação internacional na resolução da transgressão penal. Outrossim, visando reunir dados quantitativos a respeito da incidência desse tipo penal, foram analisados relatórios produzidos e publicados pela UNODC (United Nations Office on Drugs and Crimes), tais como o "Global Report on Trafficking in persons" (2020) e o "Relatório Global de Tráfico de Pessoas - Perfil de País América do Sul” (2018), os quais apresentam informações detalhadas a respeito do número de vítimas e casos detectados, dos processos e condenações reportadas e quais as finalidades de tráfico foram identificadas com maior frequência.

Posteriormente, foi analisada a questão extradicional. A princípio, coube a realização de uma análise bibliográfica e documental, visando compreender a extradição como mecanismo de cooperação internacional e seu funcionamento, dessa forma, podemos destacar as obras de J. Puente Egido (2000), Ramón J. Pena (1974), Francisco Rezek (2018) e Pedro Pablo Camargo (2000). Ademais, foram analisados os tratados bilaterais de extradição firmados entre o Brasil e os demais países da América do Sul, analisando os pré-requisitos necessários para o deferimento de um pedido extradicional, assim como as hipóteses que atuariam como proibitivas do requerimento.

Encerrado esse momento, foi inaugurada a fase empírica da pesquisa, objetivando o levantamento de casos de pedidos de extradição entre os países sul-americanos a respeito da hipótese do crime de tráfico de pessoas. Para isso, foi realizada uma busca jurisprudencial nas Cortes Supremas Internacionais de cada país da América do Sul, através da utilização de expressões de busca simples como "tráfico de pessoas", "trata de personas", "extradição", "extradicíon", ou ainda, utilizando as ferramentas de pesquisa jurisprudencial avançadas e realizando a solicitação de pesquisa através de artigos da legislação respectiva a cada nação. Buscou-se portanto, analisar os motivos que levaram à concessão e a recusa de tais pedidos, para compreender de que maneira haveria a influência do Protocolo Adicional à Convenção das Nações Unidas contra o crime organizado transnacional relativo à Prevenção, Repressão e Punição do Tráfico de Pessoas, em Especial Mulheres e Crianças no âmbito da uniformização da legislação sul-americana a respeito de tráfico de pessoas e na cooperação internacional.

\section{Resultados e Discussão}

Com base no levantamento bibliográfico realizado a respeito da tipificação do crime de tráfico de pessoas foi possível constatar que atualmente todos os países pertencentes à América do Sul, exceto a República Bolivariana da Venezuela, possuem suas legislações internas a respeito deste delito em consonância com a definição encontrada na redação do Protocolo de Palermo (UNODC, 2018). 
A definição atual do crime de tráfico de pessoas, em conformidade com o previsto no Protocolo das Nações Unidas, foi incluída no ordenamento jurídico brasileiro através da Lei $\mathrm{n}^{\circ}$ 13.344, no ano de 2016. Dessa forma, foram revogados os predecessores artigos 231 e 231-A, os quais versavam a respeito do crime de tráfico de pessoas no âmbito doméstico e internacional, contudo, era restrita à finalidade de exploração sexual. Além das restrições concernentes as elementares normativas do tipo penal, destaca-se que os dispositivos penais revogados possuíam algumas problemáticas em sua redação, como por exemplo, a utilização do termo "prostituição", como sinônimo de exploração. Tal concepção é considerada vetusta, na medida em que é perfeitamente possível que pessoas adultas, usufruindo de seu pleno consentimento, exerçam sua liberdade sexual da maneira que desejarem.

Em contrapartida, a nova tipificação penal, cuja redação encontra-se no artigo 149-A do Código Penal, buscou abranger todas as modalidades presentes no Protocolo de Palermo. Dessa forma, nos incisos I a V do artigo em questão, foram enumeradas as finalidades do delito, sendo estas a remoção de órgãos, tecidos ou partes do corpo, submissão a condições de trabalho análogas a escravidão, submissão a qualquer tipo de servidão, adoção ilegal e, por fim, a exploração sexual. O crime de tráfico de pessoas ficou definido como um crime de ação múltipla, possuindo oito núcleos do tipo penal, relativos ao agenciamento, o recrutamento, o aliciamento, o transporte, a compra, a transferência, o alojamento ou o acolhimento de pessoas. Além disso, tais condutas possuem a característica de serem alternativas e, dessa forma, mesmo que o agente pratique mais de uma dessas modalidades durante o iter criminis, será considerado um único ato criminoso contra a mesma vítima.

Existe uma situação de vulnerabilidade em volta das vítimas desse tipo criminal. Países considerados subdesenvolvidos ou em desenvolvimento costumam apresentar um perfil que favorece a incidência do tráfico de pessoas, na medida em que existe uma maior exploração de vítimas carentes, reproduzindo, dessa maneira, o modelo capitalista de exploração dos seres humanos (Borges, 2013). Ciente de tal vulnerabilidade, o Protocolo de Palermo, e consequentemente a legislação brasileira, prevê que o consentimento da vítima será considerado irrelevante nas hipóteses em que for obtido mediante fraude, coação, grave ameaça, violência ou abuso (Borges; Garbellini Filho, 2019). Nesse sentido, podemos analisar o caso Chile v. Nelly Viviana Condori Nicolas, no qual as vítimas foram contatadas através de uma agência de empregos e receberam a proposta para trabalharem como garçonetes, contudo, ao chegarem ao local estipulado, seus documentos pessoais foram confiscados e, mediante ameaças, foram obrigadas a prestar serviços sexuais (Chile, 2006).

É essencial para este estudo compreender a importância da extradição como mecanismo de cooperação internacional. Neste sentido, torna-se mister analisar alguns requisitos necessários para o deferimento de um pedido extradicional, assim como pressupostos que impedem a extradição. Para esse propósito, foi analisado o Tratado internacional firmado entre o Brasil e a Colômbia, promulgado através do Decreto nº 6.630 de 25 de Setembro de 1940 (Brasil, 1940), não havendo prejuízo em relação aos demais tratados bilaterais acordados com outros países sul-americanos, pois possuem disposições similares.

$\mathrm{O}$ tratado inicia-se proclamando que as partes contratantes obrigam-se à entrega recíproca de indivíduos, processados ou condenados pelas autoridades judiciárias, mas que encontrem-se no território da outra. Em razão dessa redação, destaca-se que, quando o pedido extradicional fundar-se em um tratado bilateral, não é possível que seja realizada a recusa sumária, a qual ocorre nas hipóteses em que o Poder Executivo nega o recebimento do pedido de maneira discricionária, de forma que sua legalidade nem chegará a ser apreciada pelo Poder Judiciário. Dessa forma, em virtude da obrigação recíproca estipulada entre os contratantes, a possibilidade de recusa sumária só pode ser exercida quando o pedido extradicional fundamentar-se em promessa de reciprocidade, sob pena de ser colocada em causa a sua responsabilidade internacional (Rezeck, 2018).

O tratado internacional estabelece a primeira hipótese de desobrigação da extradição, ou seja, quando o indivíduo for nacional do Estado requerido. Ressalta-se que tal disposição possui previsão constitucional, visto que o artigo $5^{\circ}$, inciso LI da Constituição Federal (Brasil, 1988) estabelece que nenhum brasileiro será extraditado, à exceção do brasileiro naturalizado que houver cometido um crime comum, desde que antes do processo de naturalização ou quando for comprovado o envolvimento 
com tráfico ilícito de entorpecentes. Contudo, é estipulado no Tratado bilateral que nas hipóteses em que não ocorrer a extradição do natural, o Estado requerido fica obrigado a processá-lo e julgá-lo pelo fato que lhe foi imputado, se o ato também for considerado crime (Brasil, 1940).

São apresentados outros fatores limitadores da extradição, entre eles temos a necessidade de a infração ser equivalente a um tipo penal, cuja punição seja de no mínimo um ano de prisão, assim como a necessidade do preenchimento do requisito da dupla incriminação, ou seja que o ato seja considerado delitivo tanto no país requerente, assim como no requerido. A extradição não será concedida caso o Estado requerido for considerado competente para julgar o delito, ou já estiver em andamento um processo pelo mesmo ato, ou mesmo já o tiver julgado. Além disso, não poderá ter ocorrido a prescrição da ação e são proibidas as possibilidades de apresentar o extraditando mediante um tribunal ou juízo de exceção, ou ainda, quando o delito possuir caráter puramente militar, político ou religioso (Brasil, 1940).

Nesse sentido, pode ser analisado o pedido de extradição formulado pelo Uruguai para a República da Argentina (Argentina, 2020), o qual foi negado pela Suprema Corte de Justicia de la Nación Argentina. Nesse caso concreto, o réu estaria sendo acusado de praticar o delito de tráfico de pessoas, posto que estaria realizando o recrutamento de algumas mulheres no Uruguai, sob a falsa promessa de criar uma carreira de modelo para as vítimas. Após enganá-las, as vítimas eram transportadas para as cidades de Punta del Este e Buenos Aires, e seriam exploradas sexualmente, sob o pretexto de estarem "pagando" pela fama e êxito profissional que alcançariam na carreira de modelagem. $\mathrm{O}$ caso em tela tratava de um crime transfronteiriço e as mulheres eram efetivamente exploradas em cidades argentinas, razão pela qual a Suprema Corte reconheceu a competência da Argentina para julgar o caso, de forma a indeferir o pedido formulado pela República do Uruguai. Ressalta-se que, entretanto, foi emitido o pedido ao Procurador Geral da Nação para que realizasse o julgamento do réu pelos mesmos fatos sustentados no pedido extradicional, de forma que, apesar do indeferimento, isso não implicaria na impunidade do agente (Argentina, 2020).

Em oposição à decisão da Suprema Corte argentina, é o caso da Extradição 1.290, realizada pela Colômbia ao Brasil (2013), o qual trata novamente da hipótese de tráfico de pessoas com fins de exploração sexual. A Defensoria buscou impedir a extradição do réu, alegando que, em determinado momento, algumas vítimas teriam sido transportadas para o território nacional, de maneira que o Brasil possuiria competência para julgar o caso, gerando óbice ao deferimento da extradição. Contudo, a ministra relatora, Rosa Weber, concluiu que o Brasil não possuía nenhum processo em curso a respeito do caso, nem tinha previamente julgado o réu e, dessa forma, a mera possibilidade de competência concorrente não constituiria impedimento ao pedido, deferindo a extradição.

Além da casuística apresentada, a importância decorrente da influência do Protocolo de Palermo na uniformização da legislação sul-americana a respeito do tráfico de pessoas, pode ser, por outro lado, observado no "Global Report on Trafficking in persons", que assinala que houve um aumento do percentual de condenações relativas ao crime de tráfico de pessoas na região dos países sul-americanos nos últimos 15 anos. Tal recorte temporal encontra-se em consonância com a promulgação do Protocolo do Palermo e sua gradual influência no processo de alteração das legislações internas dos países da América do Sul (UNODC, 2020).

Para demonstrar a importância de tais alterações, podemos analisar a jurisprudência colombiana na qual crianças equatorianas foram recrutadas através de um falso anúncio de emprego, com pagamentos e benefícios justos. Contudo, ao chegarem ao local combinado na República da Colômbia, foram obrigadas a trabalhar em condições desumanas e análogas à escravidão, com jornadas exaustivas, além de não receberem o salário anunciado e, ocasionalmente, nem a alimentação. Dessa forma, os réus foram acusados do crime de tráfico de pessoas. Contudo, previamente à alteração realizada através da influência do Protocolo de Palermo, apenas existia a previsão legal do delito em questão com a finalidade de exploração sexual. Sem a adequação legislativa, o caso em questão não encontraria tipificação penal no delito de tráfico de pessoas (Colômbia, 2009). 
Também é necessário delimitar a relevância do Protocolo de Palermo no âmbito das extradições. A maior influência do Protocolo das Nações Unidas ocorreu no âmbito do preenchimento do princípio da dupla incriminação ou dupla tipificação, o qual pressupõe que a conduta analisada seja considerada um delito no Estado requerente, assim como no requerido. Para isso, ressalta-se não haver necessidade de o crime receber o mesmo nomen juris em ambas as legislações (Bittencourt, 2021).

Como mencionado, de acordo com Borges (2017) e o "Global report on trafficking in persons 2020" (UNODC, 2020), apenas a República Bolivariana da Venezuela não possui a legislação interna em completa consonância com a redação a respeito do crime de tráfico de pessoas prevista no protocolo de Palermo. Por conseguinte, no que tange os demais países, o princípio da dupla incriminação sempre será preenchido, beneficiando, portanto, o deferimento das extradições. Nesse sentido, podemos analisar dois pedidos extradicionais deferidos pelo governo da Colômbia, ao Peru e ao Equador.

O requerimento formulado pela República do Equador à Corte Suprema de Justicia colombiana diz respeito a um caso concreto de tráfico de pessoas com a finalidade de exploração sexual. Durante o processo de análise da legalidade do pedido realizado pela Corte Suprema, foi apontada a existência do tratado bilateral de extradição acordado entre ambos os Estados e a necessidade do preenchimento do requisito da dupla tipificação constante no acordo. Dessa forma, foi necessário um exame com o intuito de averiguar a tipificação do delito em ambas as nações. Por fim, restou claro que o delito estaria previsto no artigo 528-11 do Código Penal equatoriano, assim como no artigo 188-A da legislação penal colombiana, ambos abarcando características similares após a incorporação do Protocolo de Palermo (Colômbia, 2016).

Outrossim, ocorreu a análise do pedido formulado pela República do Peru (Colômbia, 2015), expondo um caso concreto de tráfico de pessoas agravado. Foi realizada a mesma análise, visando o preenchimento do requisito da dupla incriminação, e foi constatada a equivalência da tipificação dos artigos 188-A e 188-B do Código Penal colombiano, com as previsões dos artigos 153 e 153-A do Código Penal peruano, culminando, portanto, no deferimento da extradição.

Apesar dos implementos e melhorias, é notável que ainda existe um descompasso entre o número de vítimas e casos de tráfico de pessoas que se tem conhecimento, e o número de pessoas processadas ou condenadas por tal crime. Neste sentido são os dados informados no Relatório Global sobre Tráfico de Pessoas - Perfil de Países da América do Sul da UNODC (United Nations Office on Drugs and Crime). Na Bolívia, foram detectadas oitocentas e oitenta vítimas de tráfico de pessoas entre 2014 e agosto de 2017, mas, em contrapartida, apenas treze pessoas foram condenadas pelo crime durante o mesmo recorte temporal.

Quando é analisado o tráfico de pessoas ou outras formas contemporâneas de trabalho escravo é importante nunca partir do pressuposto que tais crimes não existem ou possuem uma baixa incidência. Os dados desses crimes podem não ser tão perceptíveis, ainda mais quando observados com o enfoque no sujeito ativo. É uma rede criminosa extremamente furtiva e lucrativa e, muitas vezes, mesmo quando as vítimas são encontradas, a identificação dos criminosos é um trabalho árduo. Portanto, é um crime que, apesar de apresentar mudanças formais na legislação, na realidade, ainda há um longo caminho a ser combatido.

Núria Ramos Cordero e Pilar Cruz Zuñiga (2019), em sua obra, buscam tratar do tráfico de pessoas com enfoque na violação dos direitos humanos sofrida pelas vítimas. Dessa forma, elenca maneiras de combate a esse tipo criminal, mencionando que o enfrentamento ao tráfico humano necessita de uma abordagem multigarantista e multiescalar. Ou seja, uma atuação que envolva toda a sociedade, no âmbito interno e transnacional. Dessa forma, acredita que, apesar do papel principal nessa luta ser dos Estados e das instituições nacionais e internacionais, existe um dever para a sociedade civil colaborar e participar.

Exemplifica uma forma edificante de divisão de funções das instituições com o intuito de potencializar o combate e o amparo às vítimas (2019). Seria de competência das entidades públicas com apoio das entidades supranacionais a função de formulação de políticas públicas. As instruções e julgamentos do delito caberiam às entidades judiciais. Aos entes que compõem a força pública de segurança dos Estados estaria designada a tarefa de investigação e perseguição do delito, e finalmente, às organizações não-governamentais e entidades religiosas possuiriam a função de amparar a vítima. 


\section{Conclusão}

Este estudo buscou compreender a influência que o Protocolo Adicional à Convenção das Nações Unidas contra o Crime Organizado Transnacional Relativo à Prevenção, Repressão e Punição do Tráfico de Pessoas, em Especial Mulheres e Crianças poderiam apresentar em relação a facilitação das concessões de demandas extradicionais formuladas entre os países da América do Sul.

Para tanto, foi necessário compreender o funcionamento da incorporação dos tratados internacionais no ordenamento jurídico interno dos países, de modo a constatar a necessidade de alterarem a legislação penal para entrar em consonância com o Protocolo. Logo, foi possível comprovar a influência desse Tratado e sua contribuição para a homogeneização do entendimento do tráfico de pessoas entre os países sul-americanos, notadamente, em suas legislações internas.

Ainda, conclui-se que, durante a fase jurisdicional de análise dos pedidos extradicionais, o Protocolo de Palermo não é citado diretamente como força motivadora do deferimento. Contudo, ao analisarmos os pré-requisitos necessários para a concessão de uma extradição, presentes nos tratados bilaterais acordados entre os Estados, foi possível constatar a importância da homogeneização legislativa, visto que o princípio da dupla incriminação ou dupla tipicidade sempre será satisfeito.

Houve ainda uma análise a respeito de uma possível falha do sistema extradicional e a possibilidade dele ensejar conflito de competências entre os Estados requerentes e requeridos. Contudo, através da análise jurisprudencial foi constatado que mesmo nas situações envolvendo competências concorrentes, as nações buscam uma resolução pacífica do conflito, visando, primordialmente o combate ao tráfico humano e, dessa maneira, não haveria a possibilidade das criminosas usufruírem de qualquer tipo de impunidade decorrentes de conflitos de direito internacional, diante da negativa da extradição.

Finalmente, foi apurado que apesar de todos os avanços e da cooperação internacional, o crime de tráfico de pessoas ainda possui grande expressividade no mundo, em especial em países pobres e em desenvolvimento. De forma que foram elencadas algumas abordagens para o combate a esse tipo penal por Nuria Cordero e Pilar Zuñiga (2019), buscando não apenas a repressão do delito, mas primordialmente a sua prevenção, além de maior enfoque para o amparo às vítimas.

Tratando-se da possibilidade de aprofundamento do tema para futuros trabalhos, destacam-se alguns questionamentos advindos da pesquisa, os quais decerto acarretariam o aperfeiçoamento do assunto. Inicialmente, durante as pesquisas realizadas no Supremo Tribunal Federal brasileiro, foi notável a constância de extradições referentes ao crime de tráfico de pessoas deferidas para países europeus, especialmente a Espanha. O contraste numérico apresentado entre o continente europeu e o sulamericano era evidente, dessa forma, seria possível a realização de um estudo, buscando descobrir se de fato existe algum fator influenciando tal discrepância. Por exemplo, se os traficantes de pessoas, costumeiramente, refugiam-se em nações mais distantes, acreditando que dificultariam sua localização e sua extradição. Ademais, outro possível tema de aprofundamento no crime de tráfico de pessoas, poderia explorar os meios virtuais como forma de aliciamento das vítimas, especialmente devido a pandemia da Covid-19.

\section{Referências}

Argentina (2020). Corte Suprema de Justicia de La Nación Argentina.Recurso ordinário de apelação. Extradição. Votos: Maqueda, Lorenzetti, Rosatti. Data de publicação: 17 de setembro de 2020 <http://sjconsulta.csjn.gov.ar/sjconsulta/documentos/verUnicoDocumento Link.html?idAnalisis=760409\&cache=1614130789499>

Bitencourt, C.R. (2021). Tratado de Direito Penal 1- Parte Geral. Editora Saraiva. <https://integrada.minhabiblioteca.com.br/\#/books/9786555590333/>.

Borges, P. C. C. (org) (2013). Tráfico de pessoas para exploração sexual: prostituição e trabalho sexual escravo - São Paulo: NETPDH; Cultura Acadêmica Editora (Série "Tutela penal dos direitos humanos"), n. 3

Borges, P. C. C. (2017). La trata de personas como expresión de formas contemporáneas de esclavitud en América del Sur. In: Pérez-Alonso, Esteban (org). El derecho ante las formas contemporáneas de esclavitud. Valencia: Tirant lo Blanch, 369-398.

Borges, P. C. C. (2019). Legislación penal para la lucha contra la trata de personas en la región de Unasur. UAC Revista de La Faculdad de Derecho. 3 (5). 43 68. 
Borges, P. C. C.; \& Garbellini Filho, L. H. (2019). Entre construções e representações do tráfico de mulheres para prostituição, da vulnerabilidade e do consentimento: um estudo crítico sobre a aplicação da norma incriminadora pelo sistema de justiça criminal. Quaestio Juris, 12 (3), pág. 490-527. <https://www.e-publicacoes.uerj.br/index.php/quaestioiuris/article/view/39067/32698>. Acesso em: 20 de jan de 2021.

Britos, P. B. H.; \& Arturo. C. (2017). Discusión sobre los conceptos de "vulnerabilidad" y "consentimiento" en el protocolo de Palermo. Revista Jurídica Mario Alario D’Filippo, IX (18), pág 12-20.

Brasil. Decreto-Lei n ${ }^{\circ}$ 2.848, de 7 de setembro de 1940. < http://www.planalto.gov.br/ccivil_03/decreto-lei/del2848compilado.htm> Acesso em: 22 de nov de 2020

Brasil (2013). Supremo Tribunal Federal.Extradição n 1290 /DF - Distrito Federal. Relator: Ministra Rosa Weber. Pesquisa de Jurisprudência, Acórdãos, 14 de agosto de 2013. < http://redir.stf.jus.br/paginadorpub/paginador.jsp?docTP=TP\&docID=4317454 >.

Brasil (2021). Supremo Tribunal Federal. Ag.Reg. no Recurso Ordinário em Habeas Corpus $n^{\circ}$ 121.835. Relator: Ministro Celso de Mello. Pesquisa de Jurisprudência. Acórdãos, < http://redir.stf.jus.br/paginadorpub/paginador.jsp?docTP=TP\&docID=9832750 > Acesso em: 20 de mar de 2021

Brasil (1940) Decreto $\mathrm{n}^{\circ} 6.330$ de 25 de setembro de 1940. Tratado de extradição entre o Brasil e a Colômbia. http://www.justica.gov.br/suaprotecao/cooperacao-internacional/extradicao/acordos-de-extradicao-1/arquivos/decreto-no-6-330-de-25-de-setembro-de-1940.pdf..

Camargo, P. P (2000): La extradición. Revista de Derecho Penal. Universidad de Los Andes, Colombia, 17, 79-103. <https://repositorio.uniandes.edu.co/handle/1992/6093?show=full > Acesso em: 30 de jan de 2021

Colômbia (2016). Corte Suprema de Justicía República de Colombia. Extradición. Data de publicação: 8 de junho de 2016. ID: $485917 .<$ http://consultajurisprudencial.ramajudicial.gov.co:8080/WebRelatoria/csj/index.xhtml >

Colômbia (2015). Corte Suprema de Justícia República de Colombia. Extradición. Data de Publicação: 14 de outubro de 2015. ID: $447511 .<$ http://consultajurisprudencial.ramajudicial.gov.co:8080/WebRelatoria/csj/index.xhtml >.

Constituição da República Federativa do Brasil de 1988. (1998). Brasília. < http://www.planalto.gov.br/ccivil_03/constituicao/constituicao.htm >.

Cordero, N. R; Zúñiga, P. C. (2019). Trata de personas, género u migraciones en Andalucía (España), Costa Rica y Marruecos: Retos y propuestar para la defensa y garantía de los derechos humanos. DYKINSON, S.L

Egido, P. J. (2000). La extradición, problema complejo de cooperación internacional en materia penal. Boletín de La Faculdad de derecho de la UNED, Madrid. V. 15..205-236. <http://e-spacio.uned.es/fez/eserv/bibliuned:BFD-2000-15-AFB5FE53/PDF>.

Greco, R. (2019). Direito Penal Estruturado.: Grupo GEN. < https://integrada.minhabiblioteca.com.br/\#/books/9788530985875/>.

Governo Federal: Ministério da Justiça e da Segurança Pública: Departamento de Recuperação de Ativos e Cooperação Jurídica Internacional, c2020. Tráfico de Pessoas. 〈https://www.justica.gov.br/sua-protecao/trafico-de-pessoas >

Monteiro, M. A. C. (2019). Tratados Internacionais de Direitos Humanos e Direito Interno, Editora Saraiva. <https://integrada.minhabiblioteca.com.br/\#/books/9788502140448>/ . Acesso em: 31 Mar 2021

Nucci, G.S. Código Penal Comentado. Grupo GEN, 2020. < https://integrada.minhabiblioteca.com.br/\#/books/9788530990138/>.

Pellet, A. L. (2017). Lótus: quantos despropósitos são proferidos em teu nome! Considerações sobre o conceito de soberania na jurisprudência da corte mundial. Revista Publicum. v.3, n. 1. 60-77 < http://www.e-publicacoes.uerj.br/index.php/publicum >

Pena,R. J. (1974). La extradición. Revista Chilena de Derecho. Pontifícia Universidad Católica del Chile. 1(3/4). 375-380. https://www.jstor.org/stable/41605123

Rezeck, F. (2018). Direito internacional público: curso elementar. Editora Saraiva. <https://integrada.minhabiblioteca.com.br/\#/books/9788553172894/>.

Rodrigues, T. C. (2012). O Tráfico Internacional de pessoas para fim de exploração sexual e a questão do consentimento. São Paulo: Tese (Mestrado em Direito Penal), Universidade de São Paulo (USP). https://www.teses.usp.br/teses/disponiveis/2/2136/tde-30102012-102346/publico/REDAC AO_FINAL_Thais_de_Camargo_Rodrigues.pdf>.

UNODC. Escritório de ligação e parceria no Brasil, c2020. Tráfico de Pessoas e Contrabando de Migrantes. https://www.unodc.org/lpo-brazil/pt/trafico-depessoas/index.html

UNODC (2020). Global Report on Trafficking in Persons 2020. United Nations publication, Sales No. E.20.IV.3). https://www.unodc.org/documents/data-andanalysis/tip/2021/GLOTiP_2020_15jan_web.pdf

UNODC (2018). Relatório Global sobre Tráfico de Pessoas- Perfil de País América do Sul. https://www.unodc.org/documents/lpobrazil//Topics_TIP/Publicacoes/2018_GloTiP_South_America.pdf >.

UNODC. (2009). Sherloc: sharing eletronic resources and laws on crime. Colômbia. https://sherloc.unodc.org/cld/case-lawdoc/traffickingpersonscrimetype/col/2009/r.h.c.j.c.m._and_s.c.c..html?lng=en\&tmpl=sherloc >. 